\title{
Convolution estimates related to space curves
}

Youngwoo Choi

Correspondence: youngwoo@ajou. ac.kr

Department of Mathematics, Ajou University, Suwon 443-749, South Korea

\begin{abstract}
Based on a uniform estimate of convolution operators with measures on a family of plane curves, we obtain optimal $L^{p}-L^{q}$ boundedness of convolution operators with affine arclength measures supported on space curves satisfying a suitable condition. The result generalizes the previously known estimates.

2000 Mathematics Subject Classifications: Primary 42B15; Secondary 42B20.
\end{abstract}

Keywords: affine arclength, convolution operators

\section{Introduction}

Let $I \subset \mathbb{R}$ be an open interval and $\psi: I \rightarrow \mathbb{R}$ be a $C^{3}$ function. Let $\gamma: I \rightarrow \mathbb{R}^{3}$ be the curve given by $\gamma(t)=\left(t, t^{2} / 2, \psi(t)\right), t \in I$. Associated to $\gamma$ is the affine arclength measure $d \sigma_{\gamma}$ on $\mathbb{R}^{3}$ determined by

$$
\int_{\mathbb{R}^{3}} f d \sigma_{\gamma}=\int_{I} f(\gamma(t)) \lambda(t) d t, \quad f \in C_{0}^{\infty}\left(\mathbb{R}^{3}\right)
$$

with

$$
\lambda(t)=\left|\psi^{(3)}(t)\right|^{\frac{1}{6}}, \quad t \in I .
$$

The $L^{p}-L^{q}$ mapping properties of the corresponding convolution operator $T_{\sigma_{\gamma}}$ given by

$$
T_{\sigma_{\gamma}} f(x)=f * \sigma_{\gamma}(x)=\int_{I} f(x-\gamma(t)) \lambda(t) d t
$$

have been studied by many authors [1-8]. The use of the affine arclength measure was suggested by Drury [2] to mitigate the effect of degeneracy and has been helpful to obtain uniform estimates.

We denote by $\Delta$ the closed convex hull of $\left\{(0,0),(1,1),\left(p_{0}{ }^{-1}, q_{0}{ }^{-1}\right)\left(p_{1}{ }^{-1}, q_{1}{ }^{-1}\right)\right\}$ in the plane, where $p_{0}=3 / 2, q_{0}=2, p_{1}=2$ and $q_{1}=3$. The line segment joining $\left(p_{0}{ }^{-1}, q_{0}{ }^{-1}\right)$ and $\left(p_{1}{ }^{-1}, q_{1}^{-1}\right)$ is denoted by $\mathfrak{S}$. It is well known that the typeset of $T_{\sigma_{\gamma}}$ is contained in $\Delta$ and that under suitable conditions $T_{\sigma_{\gamma}}$ is bounded from $L^{p}\left(\mathbb{R}^{3}\right)$ to $L^{q}\left(\mathbb{R}^{3}\right)$ with uniform bounds whenever $\left(p^{-1}, q^{-1}\right) \in \mathfrak{S}$. The most general result currently available was obtained by Oberlin [5]. In this article, we establish uniform endpoint estimates on $T_{\sigma_{\gamma}}$ for a wider class of curves $\gamma$.

Before we state our main result, we introduce certain conditions on functions defined on intervals. For an interval $J_{1}$ in $\mathbb{R}$, a locally integrable function $\Phi: J_{1} \rightarrow \mathbb{R}^{+}$,

(c) 2011 Choi; licensee Springer. This is an Open Access article distributed under the terms of the Creative Commons Attribution License (http://creativecommons.org/licenses/by/2.0), which permits unrestricted use, distribution, and reproduction in any medium, provided the original work is properly cited. 
and a positive real number $A$, we let

$$
\begin{aligned}
\mathfrak{G}(\Phi, A):=\left\{\omega: J_{1} \rightarrow \mathbb{R}^{+} \mid \sqrt{\omega\left(s_{1}\right) \omega\left(s_{2}\right)}\right. & \leq \frac{A}{s_{2}-s_{1}} \int_{s_{1}}^{s_{2}} \Phi(s) d s \\
& \text { whenever } \left.s_{1}<s_{2} \text { and }\left[s, s_{2}\right] \subset J_{1}\right\}
\end{aligned}
$$

and

$$
\mathcal{E}_{1}(A):=\left\{\Phi: J \rightarrow \mathbb{R}^{+} \mid \Phi \in \mathfrak{G}(\Phi, A)\right\} .
$$

An interesting subclass of $\mathcal{E}_{1}(2 A)$ is the collection $\mathcal{E}_{2}(A)$, introduced in [9], of functions $\Phi: J \rightarrow \mathbb{R}^{+}$such that

1. $\Phi$ is monotone; and

2. whenever $s_{1}<s_{2}$ and $\left[s_{1}, s_{2}\right] \subset J$,

$$
\sqrt{\Phi\left(s_{1}\right) \Phi\left(s_{2}\right)} \leq A \Phi\left(\left(s_{1}+s_{2}\right) / 2\right)
$$

Our main theorem is the following:

Theorem 1.1. Let $I=(a, b) \subset \mathbb{R}$ be an open interval and let $\psi: I \rightarrow \mathbb{R}$ be a $C^{3}$ function such that

1. $\psi^{(3)}(t) \geq 0$, whenever $t \in I$;

2. there exists $A \in(0, \infty)$ such that, for each $u \in(0, b-a)$, $\mathfrak{F}_{u}:(a, b-u) \rightarrow \mathbb{R}^{+}$given by $\mathfrak{F}_{u}(s):=\sqrt{\psi^{(3)}(s+u) \psi^{(3)}(s)}$ satisfies

$$
\mathfrak{F}_{u} \in \mathcal{E}_{1}(A) .
$$

Then, the operator $T_{\sigma_{y}}$ defined by $(1.1)$ is a bounded operator from $L^{p}\left(\mathbb{R}^{3}\right)$ to $L^{q}\left(\mathbb{R}^{3}\right)$ whenever $\left(p^{-1}, q^{-1}\right) \in \mathfrak{S}$, and the operator norm $\left\|T_{\sigma_{\gamma}}\right\|_{L^{p} \rightarrow L^{q}}$ is dominated by a constant that depends only on $A$.

The case when $\psi^{(3)} \in \mathcal{E}_{2}(A)$ was considered by Oberlin [5]. One can easily see that $\psi^{(3)} \in \mathcal{E}_{2}(A / 2)$ implies (1.2) uniformly in $u \in(0, b-a)$. The theorem generalizes many results previously known for convolution estimates related to space curves, namely [1-6].

This article is organized as follows: in the following section, a uniform estimate for convolution operators with measures supported on plane curves. The proof of Theorem 1.1 based on a $T^{*} T$ method is given in Section 3.

\section{Uniform estimates on the plane}

The following theorem motivated by Oberlin [10] which is interesting in itself will be useful:

Theorem 2.1. Let $J$ be an open interval in $\mathbb{R}$, and $\varphi: J \rightarrow \mathbb{R}$ be a $C^{2}$ function such that $\varphi^{\prime \prime} \geq 0$. Let $\omega: J \rightarrow \mathbb{R}$ be a nonnegative measurable function. Suppose that there exists a positive constant $A$ such that $\omega \in \mathfrak{G}\left(\phi^{\prime \prime}, A\right)$, i.e.

$$
\omega\left(s_{1}\right)^{1 / 2} \omega\left(s_{2}\right)^{1 / 2} \leq \frac{A}{s_{2}-s_{1}} \int_{s_{1}}^{s_{2}} \phi^{\prime \prime}(v) d v
$$

holds whenever $s_{1}<s_{2}$ and $\left[s_{1}, s_{2}\right] \subset J$. Let $S$ be the operator given by

$$
\mathcal{S} g\left(x_{2}, x_{3}\right)=\int_{J} g\left(x_{2}-s, x_{3}-\phi(s)\right) \omega^{1 / 3}(s) d s
$$


for $g \in C_{0}^{\infty}\left(\mathbb{R}^{2}\right)$. Then, there exists a constant $C$ that depends only on $A$ such that

$$
\|\mathcal{S} g\|_{L^{3}\left(\mathbb{R}^{2}\right)} \leq C\|g\|_{L^{3 / 2}\left(\mathbb{R}^{2}\right)}
$$

holds uniformly in $g \in C_{0}^{\infty}\left(\mathbb{R}^{2}\right)$.

Proof of Theorem 2.1. Our proof is based on the method introduced by Drury and Guo [11], which was later refined by Oberlin [10].

We have

$$
\begin{aligned}
\|\mathcal{S} g\|_{3}^{3} & =\int_{\mathbb{R}} \int_{\mathbb{R}} \int_{J} \int_{J} \int_{J} \prod_{j=1}^{3}\left(g\left(x_{2}-s_{j}, x_{3}-\phi\left(s_{j}\right)\right) \omega^{1 / 3}\left(s_{j}\right)\right) d s_{1} d s_{2} d s_{3} d x_{2} d x_{3} \\
& =\int_{\mathbb{R}} \int_{\mathbb{R}} \int_{\mathbb{R}}\left[\mathcal{G}\left(g\left(z_{1}, \cdot\right), g\left(z_{2}, \cdot\right), g\left(z_{3}, \cdot\right)\right)\right]\left(z_{1}, z_{2}, z_{3}\right) d z_{1} d z_{2} d z_{3},
\end{aligned}
$$

where for $z_{1}, z_{2}, z_{3} \in \mathbb{R}$ and suitable functions $h_{1}, h_{2}, h_{3}$ defined on $\mathbb{R}$,

$$
\begin{array}{r}
{\left[\mathcal{G}\left(h_{1}, h_{2}, h_{3}\right)\left(z_{1}, z_{2}, z_{3}\right):=\int_{\mathbb{R}} \int_{J\left(z_{1}, z_{2}, z_{3}\right)} \prod_{j=1}^{3}\left[h_{j}\left(x_{3}-\phi\left(x_{2}-z_{j}\right)\right) \omega^{1 / 3}\left(x_{2}-z_{j}\right)\right]\right.} \\
d x_{2} d x_{3},
\end{array}
$$

and

$$
J\left(z_{1}, z_{2}, z_{3}\right):=\left(J+z_{1}\right) \cap\left(J+z_{2}\right) \cap\left(J+z_{3}\right) .
$$

We will prove that the estimate

$$
\left|\left[\mathcal{G}\left(h_{1}, h_{2}, h_{3}\right)\right]\left(z_{1}, z_{2}, z_{3}\right)\right| \leq \frac{C|| h_{1}\left\|_{L^{3 / 2}(\mathbb{R})}|| h_{2}\right\|_{L^{3 / 2}(\mathbb{R})}|| h_{3} \|_{L^{3 / 2}(\mathbb{R})}}{\left|\left(z_{1}-z_{2}\right)\left(z_{1}-z_{3}\right)\left(z_{2}-z_{3}\right)\right|^{1 / 3}}
$$

holds uniformly in $h_{1}, h_{2}, h_{3}, z_{1}, z_{2}$, and $z_{3}$.

To establish (2.1) we let

$$
\begin{aligned}
{\left[\mathcal{G}_{k}\left(h_{1}, h_{2}, h_{3}\right)\right]\left(z_{1}, z_{2}, z_{3}\right):=} & \int_{\mathbb{R}} \int_{J\left(z_{1}, z_{2}, z_{3}\right)} h_{k}\left(x_{3}-\phi\left(x_{2}-z_{k}\right)\right) \\
& \prod_{\substack{1 \leq j \leq 3 \\
j \neq k}}\left[h_{j}\left(x_{3}-\phi\left(x_{2}-z_{j}\right)\right) \omega^{1 / 2}\left(x_{2}-z_{j}\right)\right] d x_{2} d x_{3}
\end{aligned}
$$

for $k=1,2,3$. Then, we have

$$
\begin{array}{r}
\left|\left[\mathcal{G}_{1}\left(h_{1}, h_{2}, h_{3}\right)\right]\left(z_{1}, z_{2}, z_{3}\right)\right| \leq\left\|h_{1}\right\|_{\infty} \int_{\mathbb{R}} \int_{J\left(z_{1}, z_{2}, z_{3}\right)} \prod_{j=2}^{3}\left(\left|h_{j}\left(x_{3}-\phi\left(x_{2}-z_{j}\right)\right)\right| \omega^{1 / 2}\left(x_{2}-z_{j}\right)\right) \\
d x_{2} d x_{3} .
\end{array}
$$

For $z_{2}, z_{3} \in \mathbb{R}$ and $x_{2} \in J\left(z_{1}, z_{2}, z_{3}\right)$, we have

$$
\begin{aligned}
\left.\mid \phi^{\prime}\right)\left(x_{2}-z_{2}\right)-\phi^{\prime}\left(x_{2}-z_{3}\right) \mid & =\left|\int_{x_{2}-z_{2}}^{x_{2}-z_{3}} \phi^{\prime \prime}(s) d s\right| \\
& \geq A^{-1}\left|z_{2}-z_{3}\right| \omega^{1 / 2}\left(x_{2}-z_{2}\right) \omega^{1 / 2}\left(x_{2}-z_{3}\right)
\end{aligned}
$$

by hypothesis. Hence,

$$
\begin{aligned}
\left|\left[\mathcal{G}_{1}\left(h_{1}, h_{2}, h_{3}\right)\right]\left(z_{1}, z_{2}, z_{3}\right)\right| \leq \frac{A|| h_{1} \|_{\infty}}{\left|z_{2}-z_{3}\right|} \int_{\mathbb{R}} \int_{J\left(z_{1}, z_{2}, z_{3}\right)} \prod_{j=2}^{3}\left|h_{j}\left(x_{3}-\phi\left(x_{2}-z_{j}\right)\right)\right| & \left|\phi^{\prime}\left(x_{2}-z_{2}\right)-\phi^{\prime}\left(x_{2}-z_{3}\right)\right| d x_{2} d x_{3} .
\end{aligned}
$$


A change of variables gives

$$
\left|\left[\mathcal{G}_{1}\left(h_{1}, h_{2}, h_{3}\right)\right]\left(z_{1}, z_{2}, z_{3}\right)\right| \leq \frac{A|| h_{1} \|_{\infty}}{\left|z_{2}-z_{3}\right|} \int_{\mathbb{R}} \int_{\mathbb{R}}\left|h_{2}\left(z_{2}\right)\right|\left|h_{3}\left(z_{3}\right)\right| d z_{2} d z_{3} .
$$

Thus, we obtain

$$
\left|\left[\mathcal{G}_{1}\left(h_{1}, h_{2}, h_{3}\right)\right]\left(z_{1}, z_{2}, z_{3}\right)\right| \leq \frac{A|| h_{1}||_{\infty}\left\|h_{2}\right\|_{1}|| h_{3} \|_{1}}{\left|z_{2}-z_{3}\right|} .
$$

Similarly, we get

$$
\left|\left[\mathcal{G}_{2}\left(h_{1}, h_{2}, h_{3}\right)\right]\left(z_{1}, z_{2}, z_{3}\right)\right| \leq \frac{A|| h_{1}\|\|_{1}\left\|h_{2}\right\|_{\infty}|| h_{3} \|_{1}}{\left|z_{1}-z_{3}\right|}
$$

and

$$
\left|\left[\mathcal{G}_{2}\left(h_{1}, h_{2}, h_{3}\right)\right]\left(z_{1}, z_{2}, z_{3}\right)\right| \leq \frac{A|| h_{1}\left|\left\|_ { 1 } | | h _ { 2 } \left|\left\|_{1}|| h_{3}\right\|_{\infty}\right.\right.\right.}{\left|z_{1}-z_{2}\right|} .
$$

Interpolating (2.2), (2.3) and (2.4) provides (2.1). Combining this with Proposition 2.2 in Christ [12] finishes the proof.

The special case in which $\omega=\varphi^{\prime \prime}$ provides a uniform estimate for the convolution operators with affine arclength measure on plane curves.

Corollary 2.2. Let $J$ be an open interval in $\mathbb{R}$, and $\varphi: J \rightarrow \mathbb{R}$ be a $C^{2}$ function such that $\varphi^{\prime \prime} \geq 0$. Suppose that there exists a constant $A$ such that $\phi^{\prime \prime} \in \mathcal{E}_{1}(A)$, i.e.

$$
\phi^{\prime \prime}\left(s_{1}\right)^{1 / 2} \phi^{\prime \prime}\left(s_{2}\right)^{1 / 2} \leq \frac{A}{s_{2}-s_{1}} \int_{s_{1}}^{s_{2}} \phi^{\prime \prime}(v) d v
$$

holds whenever $s_{1}<s_{2}$ and $\left[s_{1}, s_{2}\right] \subset J$. Let Sbe the operator given by

$$
\mathcal{S} g\left(x_{2}, x_{3}\right)=\int_{J} g\left(x_{2}-s, x_{3}-\phi(s)\right) \phi^{\prime \prime}(s)^{1 / 3} d s
$$

for $g \in C_{0}^{\infty}\left(\mathbb{R}^{2}\right)$. Then, there exists a constant $C$ that depends only on $A$ such that

$$
\|\mathcal{S} g\|_{L^{3}\left(\mathbb{R}^{2}\right)} \leq C\|g\|_{L^{3 / 2}\left(\mathbb{R}^{2}\right)}
$$

holds uniformly in $g \in C_{0}^{\infty}\left(\mathbb{R}^{2}\right)$.

\section{Proof of the main theorem}

Before we proceed the proof of Theorem 1.1, we note that the uniform estimate (1.2) in $u \in(0, b-a)$ implies

$$
\psi^{(3)} \in \mathcal{E}_{1}(A)
$$

by continuity of $\psi^{(3)}$.

By duality and interpolation, it suffices to show that

$$
\left\|T_{\sigma_{\gamma}} f\right\|_{L^{2}\left(\mathbb{R}^{3}\right)} \leq C|| f \|_{L^{3 / 2}\left(\mathbb{R}^{3}\right)}
$$

holds uniformly for $f \in L^{3 / 2}\left(\mathbb{R}^{3}\right)$.

Recall the following lemma observed by Oberlin [3]:

Lemma 3.1. Suppose there exists a constant $C_{1}$ such that

$$
\left\|T_{\sigma_{\gamma}}^{*} T_{\sigma_{\gamma}} f\right\|_{L^{3}\left(\mathbb{R}^{3}\right)} \leq C_{1}\|f\|_{L^{3 / 2}\left(\mathbb{R}^{3}\right)}
$$

holds uniformly in $f \in L^{3 / 2}\left(\mathbb{R}^{3}\right)$. Then, (3.2) holds for each $f \in L^{3 / 2}\left(\mathbb{R}^{3}\right)$. 
To establish (3.3), we write

$$
\begin{gathered}
T_{\sigma_{\gamma}}^{*} T_{\sigma_{\gamma}} f(x)=\int_{I} \int_{I} f(x-\gamma(t)+\gamma(s)) \lambda(t) \lambda(s) d t d s \\
\text { equiv } \mathcal{T}^{(1)} f(x)+\mathcal{T}^{(2)} f(x),
\end{gathered}
$$

where

$$
\begin{aligned}
& \mathcal{T}^{(1)} f(x)=\iint_{\substack{t, s \in I \\
t>s}} f(x-\gamma(t)+\gamma(s)) \lambda(t) \lambda(s) d t d s, \\
& \mathcal{T}^{(2)} f(x)=\iint_{\substack{t, s \in I \\
t<s}} f(x-\gamma(t)+\gamma(s)) \lambda(t) \lambda(s) d t d s .
\end{aligned}
$$

By symmetry, it suffices to prove

$$
\left\|\mathcal{T}^{(1)} f\right\|_{L^{3}\left(\mathbb{R}^{3}\right)} \leq C_{1}\|f\|_{L^{3 / 2}\left(\mathbb{R}^{3}\right)} .
$$

Next we make a change of variables, $u=t-s$ and write for $u \in(0, b-a)$

$$
\begin{aligned}
I_{u} & =\{s \in \mathbb{R}: a<s<b-u\}, \\
\Psi_{u}(s) & =\psi(s+u)-\psi(s) .
\end{aligned}
$$

Then, we obtain:

$$
\begin{aligned}
\mathcal{T}^{(1)} f(x) & =\int_{I} \int_{0}^{b-s} f\left(x_{1}-u, x_{2}-u(s+u / 2), x_{3}-\Psi_{u}(s)\right) \lambda(s+u) \lambda(s) d u d s \\
& =\int_{0}^{b-a} \int_{I_{u}} f\left(x_{1}-u, x_{2}-u(s+u / 2), x_{3}-\Psi_{u}(s)\right) \lambda(s+u) \lambda(s) d s d u,
\end{aligned}
$$

and so

$$
\mathcal{T}^{(1)} f\left(x_{1}, x_{2}, x_{3}\right)=\int_{0}^{b-a} \mathcal{T}_{u}\left[f_{u}\left(x_{1}-u, \cdot, \cdot \cdot\right)\right]\left(\left(x_{2}-u^{2} / 2\right) / u, x_{3}\right) \frac{d u}{u^{2 / 3}}
$$

where

$$
\begin{aligned}
f_{u}\left(x_{1}, x_{2}, x_{3}\right) & :=u^{1 / 3} f\left(x_{1}, u x_{2}, x_{3}\right) \\
\mathcal{T}_{u} g\left(x_{2}, x_{3}\right) & :=\int_{I_{u}} g\left(x_{2}-s, x_{3}-\Psi_{u}(s)\right) \Lambda_{u}^{1 / 3}(s) d s \\
\Lambda_{u}(s) & :=u \lambda^{3}(s+u) \lambda^{3}(s) \\
& =u \sqrt{\psi^{(3)}(s+u) \psi^{(3)}(s)}
\end{aligned}
$$

for $x_{1}, x_{2}, x_{3} \in \mathbb{R}, u \in(0, b-a), s \in I_{u}$.

Notice that for $u \in(0, b-a)$ and $\left[s_{1}, s_{2}\right] \subset I_{u}$, we have

$$
\begin{aligned}
\Lambda_{u}^{1 / 2}\left(s_{1}\right) \Lambda_{u}^{1 / 2}\left(s_{2}\right) & \leq \frac{A u}{s_{2}-s_{1}} \int_{s_{1}}^{s_{2}} \sqrt{\psi^{(3)}(s+u) \psi^{(3)}(s) d s} \\
& \leq \frac{A^{2} u}{s_{2}-s_{1}} \int_{s_{1}}^{s_{2}} \frac{1}{u} \int_{s}^{s+u} \psi^{(3)}(v) d v d s \\
& =\frac{A^{2}}{s_{2}-s_{1}} \int_{s_{1}}^{s_{2}}\left(\psi^{\prime \prime}(s+u)-\psi^{\prime \prime}(s)\right) d s \\
& =\frac{A^{2}}{s_{2}-s_{1}} \int_{s_{1}}^{s_{2}} \Psi^{\prime \prime}{ }_{u}(s) d s
\end{aligned}
$$


by (1.2) and (3.1). By Theorem 2.1, $\left\|\mathcal{T}_{u}\right\|_{L^{3 / 2}\left(\mathbb{R}^{2}\right) \rightarrow L^{3}\left(\mathbb{R}^{2}\right)}$ is uniformly bounded. Hence, we obtain

$$
\begin{aligned}
\left\|\mathcal{T}^{(1)} f\right\|_{3} & \leq\left(\int_{\mathbb{R}}\left[\iint_{\mathbb{R}^{2}}\left(\int_{0}^{b-a}\left|\mathcal{T}_{u} f_{u}\left(x_{1}-u, \cdot, \cdot \cdot\right)\left(\frac{x_{2}-u^{2} / 2}{u}, x_{3}\right)\right| \frac{d u}{u^{2 / 3}}\right)^{3} d x_{2} d x_{3}\right]^{\frac{1}{3} \cdot 3} d x_{1}\right)^{\frac{1}{3}} \\
& \leq\left(\int_{\mathbb{R}}\left[\int_{0}^{b-a}\left(\iint_{\mathbb{R}^{2}}\left|\mathcal{T}_{u} f_{u}\left(x_{1}-u, \cdot, \cdot \cdot\right)\left(\frac{x_{2}-u^{2} / 2}{u}, x_{3}\right)\right|^{3} d x_{2} d x_{3}\right)^{\frac{1}{3}} \frac{d u}{u^{2 / 3}}\right]^{3} d x_{1}\right)^{\frac{1}{3}} \\
& \leq C(A)\left(\int_{\mathbb{R}}\left[\int_{0}^{b-a} u^{\frac{1}{3}}\left\|f_{u}\left(x_{1}-u, \cdot, \cdot \cdot\right)\right\|_{L^{3 / 2}\left(\mathbb{R}^{2}\right)} \frac{d u}{u^{2 / 3}}\right]^{3} d x_{1}\right)^{\frac{1}{3}} \\
& \leq C(A)\left(\int_{\mathbb{R}}\left[\int_{0}^{b-a}\left\|f\left(x_{1}-u, \cdot, \cdot \cdot\right)\right\|_{L^{3 / 2}\left(\mathbb{R}^{2}\right)} \frac{d u}{u^{2 / 3}}\right]^{3} d x_{1}\right)^{\frac{1}{3}} .
\end{aligned}
$$

By Hardy-Littlewood-Sobolev theorem on fractional integration, we obtain

$$
\left\|\mathcal{T}^{(1)} f\right\|_{3} \leq C_{1}(A)\|f\|_{3 / 2}
$$

This finishes the proof of Theorem 1.1.

\section{Competing interests}

The author declares that they have no competing interests.

Received: 27 April 2011 Accepted: 25 October 2011 Published: 25 October 2011

\section{References}

1. Choi, Y: The $L^{p}-L^{q}$ mapping properties of convolution operators with the affine arclength measure on space curves. J Aust Math Soc. 75, 247-261 (2003). doi:10.1017/S144678870000375X

2. Drury, SW: Degenerate curves and harmonic analysis. Math Proc Cambridge Philos Soc. 108, $89-96$ (1990). doi:10.1017/ S0305004100068973

3. Oberlin, DM: Convolution estimates for some measures on curves. Proc Am Math Soc. 99, 56-60 (1987). doi:10.1090/ S0002-9939-1987-0866429-6

4. Oberlin, DM: Convolutions with measures on curves in $\mathbb{Z}^{3}$. Can Math Bull. 41, 478-480 (1998). doi:10.4153/CMB-1998$062-7$

5. Oberlin, DM: Convolution with measures on flat curves in low dimensions. J Funct Anal. 259, 1799-1815 (2010) doi:10.1016/j.jfa.2010.05.008

6. Pan, $Y: L^{P}$-improving properties for some measures supported on curves. Math Scand. 73, $215-228$ (1993)

7. Pan, Y: Convolution estimates for some degenerate curves. Math Proc Cambridge Philos Soc. 116, 143-146 (1994). doi:10.1017/S0305004100072431

8. Secco, S: Fractional integration along homogeneous curves in $\mathbb{X}^{3}$. Math Scand. 85, 259-270 (1999)

9. Bak, JG, Oberlin, D, Seeger, A: Restriction of Fourier transforms to curves. II. Some classes with vanishing torsion. J Aust Math Soc. 85, 1-28 (2008). doi:10.1017/S1446788708000578

10. Oberlin, DM: Two estimates for curves in the plane. Proc Am Math Soc. 132, 3195-3201 (2004). doi:10.1090/S0002-993904-07610-5

11. Drury, SW, Guo, K: Convolution estimates related to surfaces of half the ambien dimension. Math Proc Cambridge Philos Soc. 110, 151-159 (1991). doi:10.1017/S0305004100070201

12. Christ, M: On the restriction of the Fourier transform to curves: endpoint results and the degenerated case. Trans Am Math Soc. 287, 223-238 (1985). doi:10.1090/S0002-9947-1985-0766216-6

doi:10.1186/1029-242X-2011-91

Cite this article as: Choi: Convolution estimates related to space curves. Journal of Inequalities and Applications 2011 2011:91. 